\title{
Biocompatibility of Thermally Hydrocarbonized Porous Silicon Nanoparticles and their Biodistribution in Rats
}

\author{
Luis M. Bimbo, ${ }^{\dagger, \|}$ Mirkka Sarparanta, ${ }^{\ddagger}, \|$ Hélder A. Santos, ${ }^{+{ }^{+*}}$ Anu J. Airaksinen, ${ }^{*}$ Ermei Mäkilä, ${ }^{\$}$ \\ Timo Laaksonen, ${ }^{\dagger}$ Leena Peltonen, ${ }^{+}$Vesa-Pekka Lehto, ${ }^{\perp}$ Jouni Hirvonen, $^{+}$and Jarno Salonen ${ }^{\mathcal{S}_{*} *}$ \\ ${ }^{\dagger}$ Division of Pharmaceutical Technology, Faculty of Pharmacy, University of Helsinki, Fl-00014 Finland, `Laboratory of Radiochemistry, Department of Chemistry, \\ University of Helsinki, Fl-00014 Finland, 'aboratory of Industrial Physics, Department of Physics, University of Turku, FI-20014 Finland, and ${ }^{\perp}$ Department of Physics, \\ University of Kuopio, Fl-70211 Kuopio, Finland. "These authors contributed equally to this work.
}

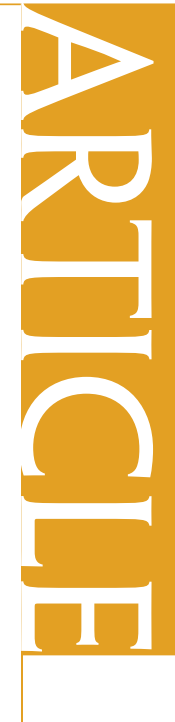

$S$ ince the first evidence of the biocompatibility of porous silicon (PSi), ${ }^{1}$ there have been a growing number of publications about its numerous applications. ${ }^{2-4}$ While most of the research has been focused on microdevices for therapeutic use, ${ }^{5}$ chemical and biological sensors, ${ }^{6}$ and optoelectronics, ${ }^{7}$ other applications have also emerged, such as intravenous $^{8}$ and ocular drug delivery. ${ }^{9,10}$ Oral delivery still remains the route preferred by patients and thus dominates controlled release research. ${ }^{11}$ Drug and peptide delivery by PSi particles has already been demonstrated and with promising results. ${ }^{12,13} \mathrm{PSi}$ particles can be loaded with diverse payloads, such as small molecule drugs and peptides. In addition, improved solubility of poorly soluble drugs after loading has been reported..$^{14-16}$ The silicon surface can be treated in the gas phase by oxidation, hydrosilylation, and thermal carbonization, ${ }^{3}$ or it can also be grafted with numerous functional moieties, including polyethyleneglycol ${ }^{17}$ and folate. ${ }^{18}$ Recently, the suitability of thermally hydrocarbonized silicon (THCPSi) microparticles for peptide delivery was demonstrated. ${ }^{19}$ The stabilization of PSi with thermal carbonization was used to prevent chemical reactions between the peptide and the PSi surface. Despite the stability, further labeling or functionalization of the surface is still feasible. Microparticles did not induce changes in plasma cytokine concentrations after subcutaneous administration in rats. ${ }^{19}$ By combining the advantageous properties of the PSi materials with those of nanoscaled vehicles, even greater

ABSTRACT Porous silicon (PSi) particles have been studied for the effects they elicit in Caco-2 and RAW 264.7 macrophage cells in terms of toxicity, oxidative stress, and inflammatory response. The most suitable particles were then functionalized with a novel ${ }^{18} \mathrm{~F}$ label to assess their biodistribution after enteral and parenteral administration in a rat model. The results show that thermally hydrocarbonized porous silicon (THCPSi) nanoparticles did not induce any significant toxicity, oxidative stress, or inflammatory response in Caco-2 and RAW 264.7 macrophage cells. Fluorescently labeled nanoparticles were associated with the cells surface but were not extensively internalized. Biodistribution studies in rats using novel ${ }^{18} \mathrm{~F}$-labeled THCPSi nanoparticles demonstrated that the particles passed intact through the gastrointestinal tract after oral administration and were also not absorbed from a subcutaneous deposit. After intravenous administration, the particles were found mainly in the liver and spleen, indicating rapid removal from the circulation. Overall, these silicon-based nanosystems exhibit excellent in vivo stability, low cytotoxicity, and nonimmunogenic profiles, ideal for oral drug delivery purposes.

KEYWORDS: porous silicon - nanoparticles · oral delivery · cytotoxicity · biodistribution - Caco-2 cells

benefits in the development of drug delivery carriers can be achieved. ${ }^{8}$ It is known that silicon-based nanomaterials can degrade rapidly in vivo into orthosilicic acid and therefore be easily excreted from the animal body. ${ }^{8}$ However, limited attention has been drawn to the toxicological profiling of the PSi degradation products. The inherent luminescence of oxidized PSi materi$\mathrm{als}^{20}$ is advantageous when noninvasive imaging is considered. ${ }^{8}$ Nonetheless, not all modified PSi materials exhibit luminescence and, therefore, alternative labeling approaches need to be investigated to assess their performance noninvasively in vivo. The use of optical imaging techniques is essentially limited to mice due to the limited light penetration depth, rendering

\section{*Address correspondence to jarno.salonen@utu.fi, helder.santos@helsinki.fi.}

Received for review November 19, 2009 and accepted May 20, 2010.

Published online May 28, 2010. 10.1021/nn901657w

() 2010 American Chemical Society 


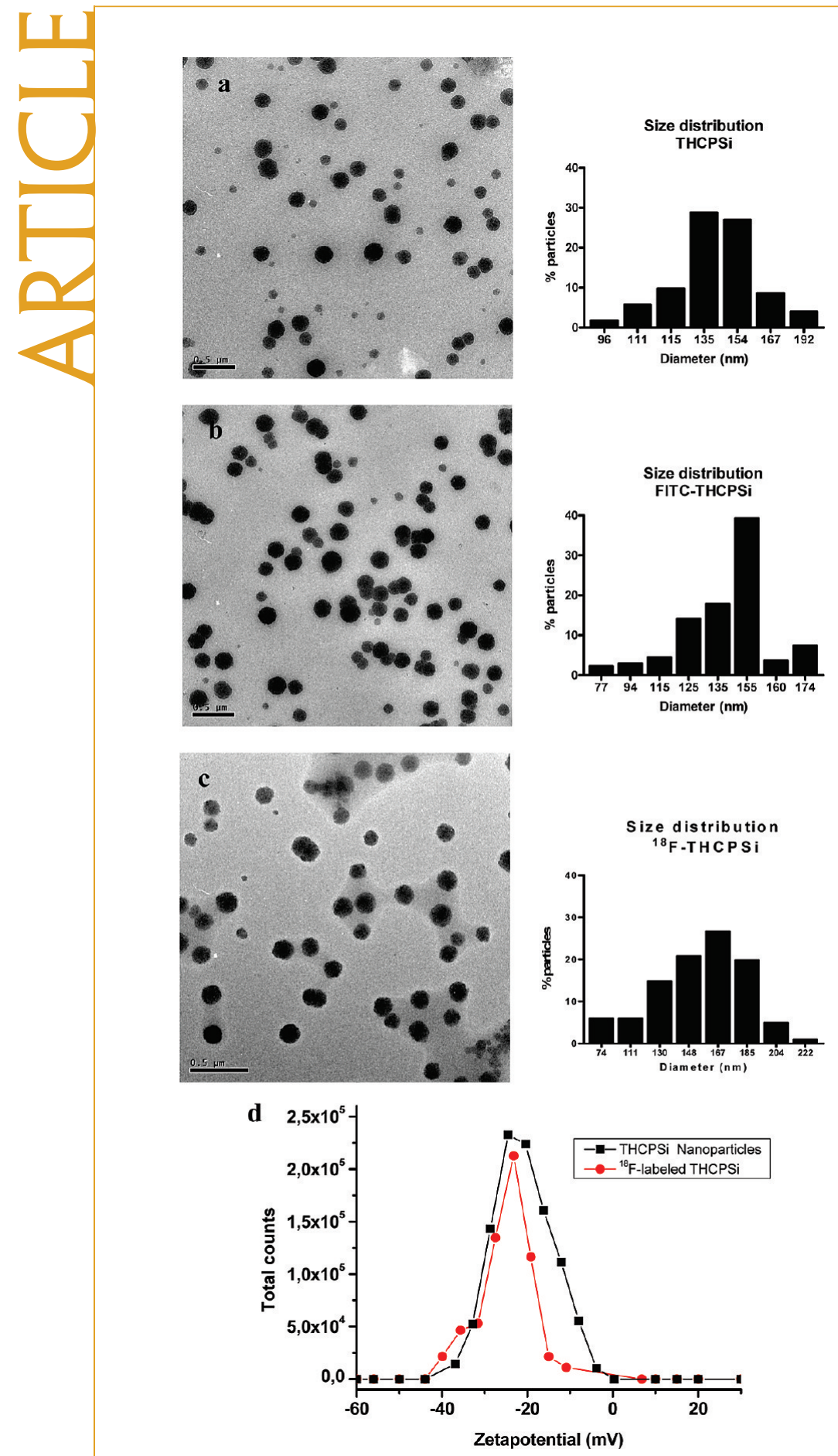

Figure 1. Transmission electron microscope (TEM) images of unlabeled THCPSi (a), FITC-THCPSi labeled (b), and ${ }^{18} \mathrm{~F}$-THCPSi labeled (c) nanoparticles used in this study and their respective size distribution. Scale bars are $500 \mathrm{~nm}$. Measured potentials of THCPSi nanoparticles (d) before labeling (squares) and after ${ }^{18} \mathrm{~F}$-labeling (circles).

nuclear imaging the only method fully translational from laboratory animals to humans.

When the potential of novel biomaterials or nanomaterials is considered, a systematic investigative approach is required to evaluate their potential hazard upon administration. ${ }^{21}$ The particle size is a key element to be taken into account, as the surface area of the particles in contact with the cell varies. For evaluation of

biocompatibility and suitability of THCPSi nanoparticles for oral delivery applications, different particle sizes were investigated. They were then tested with both Caco-2 human colon carcinoma (an in vitro model for the intestinal epithelium barrier) and RAW $264.7 \mathrm{mu}$ rine macrophage cells (an in vitro model used for cellular uptake and inflammation studies), and their biodistribution in rats was determined. Here we demonstrate that the THCPSi nanoparticles have low toxicity, do not elicit significant oxidative or inflammatory response, and associate strongly with the cell membrane without internalizing. Furthermore, radiolabeled THCPSi nanoparticles pass intact through the gastrointestinal tract and do not permeate the intestinal wall. From the results drawn in this study, we conclude that THCPSi nanoparticles are a safe and promising vehicle for oral drug delivery applications.

\section{RESULTS AND DISCUSSION}

Production and Characterization of Porous Silicon Particles. Porous Si films were synthesized by electrochemical etching the Si wafers with HF and ethanol. To perform the in vitro and in vivo studies, we have prepared thermally hydrocarbonized silicon (THCPSi) particles with several sizes ranging from micro- to nanosize. The preparation and physicochemical characterization of the PSi microparticles have been described in detail in the literature. $3,12,14,15,19,22$ In this study, two microsized THCPSi fractions were prepared: $1-10$ and $10-25 \mu \mathrm{m}$. The average pore diameter of these particles was $9.8 \mathrm{~nm}$. The specific surface area was $322 \mathrm{~m}^{2} / \mathrm{g}$, and the pore volume was $0.81 \mathrm{~cm}^{3} / \mathrm{g}$. In the case of PSi nanoparticles, the fractions prepared were 97,142 , and $188 \mathrm{~nm}$. The average pore diameter of the THCPSi nanoparticles was $9.0 \mathrm{~nm}$. The specific surface area was $202 \mathrm{~m}^{2} / \mathrm{g}$, and the pore volume was $0.51 \mathrm{~cm}^{3} / \mathrm{g}$. The measured zeta $(\zeta)$-potential of the THCPSi nanoparticles was about $-30 \mathrm{mV}$.

After etching and drying, the PSi surfaces are unstable in aqueous solution due to oxidation of the reactive surface hydrides, and therefore, a thermal hydrocarbonization was used to improve its stability. The particles obtained in this manner are stable and allow further surface modifications with molecules such as fluorescent labels or radioisotopes.

Figure 1a shows the transmission electron microscope (TEM) pictures of THCPSi nanoparticles prepared by the above-mentioned method. The particles showed a very spherical-round shape with an average size of 142 $\mathrm{nm}$. The surface of the THCPSi nanoparticles was further modified with fluorescein isothiocyanate (FITC) for cell association and uptake studies (Figure 1b). These particles exhibited similar size as the $142 \mathrm{~nm}$ unlabeled THCPSi (Figure 1a,b). In addition, the THCPSi nanoparticles were also grafted with ${ }^{18} \mathrm{~F}$ for the in vivo studies (Figure $1 \mathrm{c}$, see Materials and Methods for details). 
The specific radioactivity of cyclotron-produced $\left[{ }^{18} \mathrm{~F}\right]$ fluoride is high, typically exceeding $5 \mathrm{Ci} / \mu \mathrm{mol}(185$ $\mathrm{GBq} / \mu \mathrm{mol}) .{ }^{23}$ In this study, $0.5-1.0 \mathrm{GBq}$ of no-carrier added $\left[{ }^{18} \mathrm{~F}\right]$ fluoride corresponding to less than $0.1 \mu \mathrm{g}$ in mass was used to label a 10000-fold excess of $1 \mathrm{mg}$ of THCPSi nanoparticles. Therefore, radiolabeling of the THCPSi surface with a trace amount of ${ }^{18} \mathrm{~F}$ is unlikely to result in detectable change in the particle size and surface chemistry and, consequently, alterations in the in vivo behavior of the nanoparticles. Most importantly, it can be seen that the labeling of the THCPSi surface with FITC or ${ }^{18} \mathrm{~F}$ did not affect the physicochemical properties of the particles. All the labeled particles were morphologically similar to unlabeled THCPSi nanoparticles in terms of size and shape (Figure $1 \mathrm{a}-\mathrm{c}$ ), as well as in terms of surface charge, as demonstrated by the negative $\zeta$-potential (Figure 1d). Furthermore, the lack of influence of ${ }^{18} \mathrm{~F}$ radiolabeling on the surface chemistry was confirmed by the identical FTIR spectra of both unlabeled and radiolabeled THCPSi free-standing films (see Supporting Information, Figure 1).

In Vitro Toxicity, ROS Production, and Inflammatory Reaction. We evaluated the in vitro cytotoxic, oxidative, and inflammatory responses in the above-mentioned cell lines when exposed to the THCPSi particles for $24 \mathrm{~h}$. PSi particles are known to react in common colorimetric viability assays such as the $\mathrm{MTT}^{24}$ or $\mathrm{LDH}$, leading to false results. Therefore, a validated luminescent method based on the quantification of metabolically active (in terms of ATP content) cells was used..$^{25}$ The THCPSi microparticles of size fraction 1-10 $\mu \mathrm{m}$ were found to be more cytotoxic than all other particle sizes in the Caco- 2 cell line, although the 10-25 $\mu \mathrm{m}$ particles exhibited also some toxicity at higher concentrations (Figure 2a). THCPSi nanoparticles with an average size of 97 and $188 \mathrm{~nm}$ reduced viability compared to $142 \mathrm{~nm}$ particles in RAW 264.7 macrophages (Figure $2 \mathrm{~b}$ ). The intracellular reactive oxygen species (ROS) production, which is often used to evaluate nanoparticle toxicity ${ }^{21}$ was assessed using dichlorodihydrofluorescein diacetate (DCF-DA). This probe is nonfluorescent unless oxidized by the intracellular ROS to fluorescent DCF. The extent of oxidative response, evaluated in terms of the intracellular ROS, was of the same order of magnitude throughout all size ranges studied for both cell cultures and significantly lower than for the positive control, $\mathrm{H}_{2} \mathrm{O}_{2}$ (Figure 2c,d). The THCPSi microparticleinduced intracellular ROS production remained low despite the lower viability values, which suggests that these particles are interacting with the extracellular cell membranes, resulting in cellular damage due to ATP depletion without further production of ROS. Inflammatory responses were measured in terms of tumor necrosis factor $\alpha$ (TNF- $\alpha$ ) production. TNF- $\alpha$ is a polypeptide cytokine produced by macrophages that stimulates acute phase reaction. ${ }^{26}$ The results demonstrated that THCPSi nanoparticles with an average size of 142 and $188 \mathrm{~nm}$ triggered a lower production of TNF- $\alpha$ than the other in- vestigated particles (Figure 2e). These results are in good agreement with those shown in Figure $2 a-d$.

It has been shown that particle size plays a crucial role in determining cytotoxicity, as different pathways and cellular processes are engaged and modulated upon particle contact. ${ }^{27,28}$ Our results suggest that the most toxic size fraction of THCPSi particles is $1-10 \mu \mathrm{m}$, which reduced the cell viability the most (Figure $2 a, b$ ). On the other hand, the THCPSi particles of $142 \mathrm{~nm}$ interacted less strongly with the cells, thus, inducing less cellular damage and maintaining high levels of cellular ATP production even after $24 \mathrm{~h}$. These effects were both size and concentration dependent. It is already established that particle size can strongly affect the efficiency of cellular uptake 28,29 and there seems to be a correlation between several size ranges and a significant alteration in signaling processes. ${ }^{27}$ The fact that different internalization pathways can occur even between particles of a narrow range of sizes and also that due to the fact that most microand nanoparticle dispersions are not completely homogeneous accounts for the possibility that minor fractions in particle dispersions could be responsible for some of the biological effects. These results illustrate the importance of a clear understanding and control of particle size distribution in micro- and nanoparticle dispersions for biological purposes. The route of internalization into the cell, as well as the interaction with the cell wall, can extensively modulate viability. Inorganic particles below 100 $\mathrm{nm}$ were found to be internalized to a greater extent and to reduce viability. ${ }^{25,27}$ However, it has also been suggested that an inflection point on the particular internalization route is apparent at a size of approximately 200 $\mathrm{nm},{ }^{28}$ which is also corroborated in our study. It is for that reason that when designing a micro- or nanoparticulate drug delivery system it is advisible to avoid the thresholds that were found to have a greater impact on cell viability. In RAW 264.7 macrophages, the oxidative and cytotoxic responses seem to be of a higher level when compared to Caco-2 cells, which can be attributed to their role in the inflammatory process in response to the particles. ${ }^{30}$ The cytotoxicity and oxidative stress induced by silicic acid resulting from the complete dissolution of the $\mathrm{TH}$ CPSi particles was also investigated. Silicic acid from the dissolved particles was noncytotoxic and did not elicit any oxidative response in Caco-2 cells at concentrations up to $100 \mu \mathrm{M}$ (see Supporting Information, Figure 2, and also details on the silicic acid determination).

In Vitro Cell Association. Because the THCPSi nanoparticles of $142 \mathrm{~nm}$ revealed the most promising results in both Caco-2 and RAW 264.7 macrophage cells, we proceeded to further characterize the in vitro cellular association of these nanoparticles in detail. For this purpose, the Caco-2 and RAW 264.7 macrophage cells were incubated with the FITC labeled THCPSi nanoparticles. These particles showed a strong cellular association in a concentration-dependent manner (Figure 3a,b). 


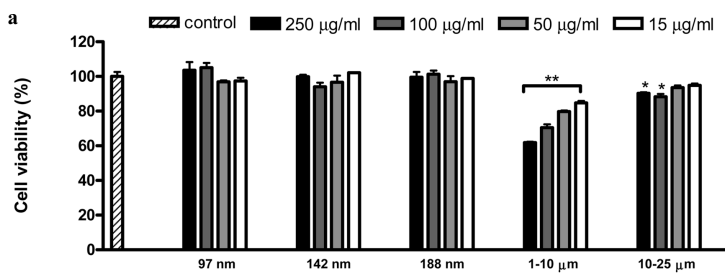

b control $250 \mu \mathrm{g} / \mathrm{ml} \square 100 \mu \mathrm{g} / \mathrm{ml} \square 50 \mu \mathrm{g} / \mathrm{ml}$ Ш $15 \mu \mathrm{g} / \mathrm{ml}$
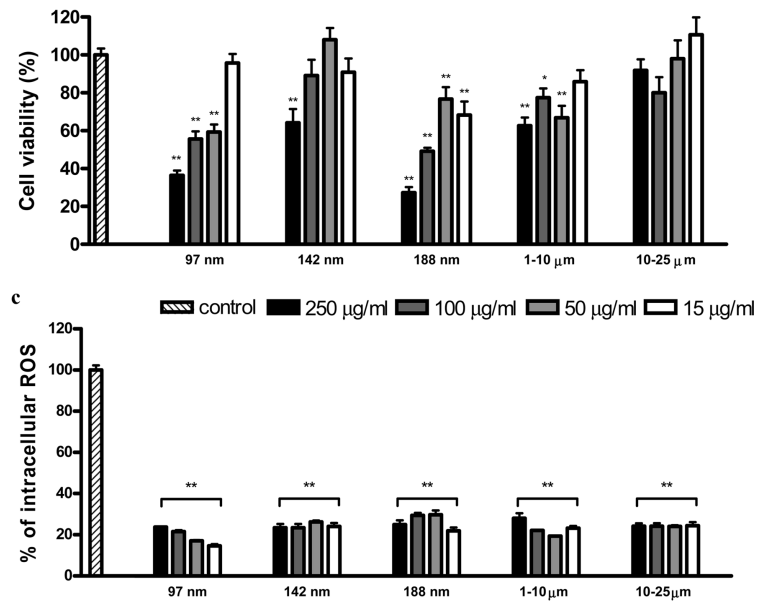

d control $250 \mu \mathrm{g} / \mathrm{ml} \square 100 \mu \mathrm{g} / \mathrm{ml} \square 50 \mu \mathrm{g} / \mathrm{ml}$ 口 $\square 15 \mu \mathrm{g} / \mathrm{ml}$

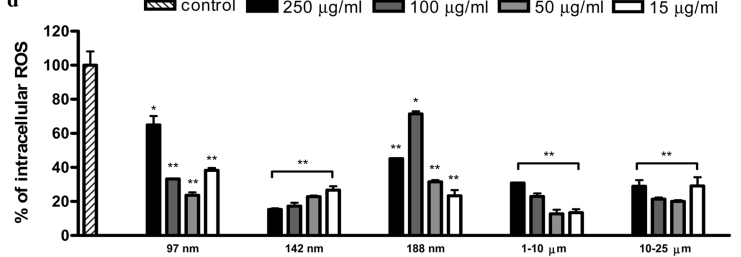

e $\quad 250 \mu \mathrm{g} / \mathrm{ml}$ Control $\square 100 \mu \mathrm{g} / \mathrm{ml} \square 50 \mu \mathrm{g} / \mathrm{ml} \square 15 \mu \mathrm{g} / \mathrm{ml}$

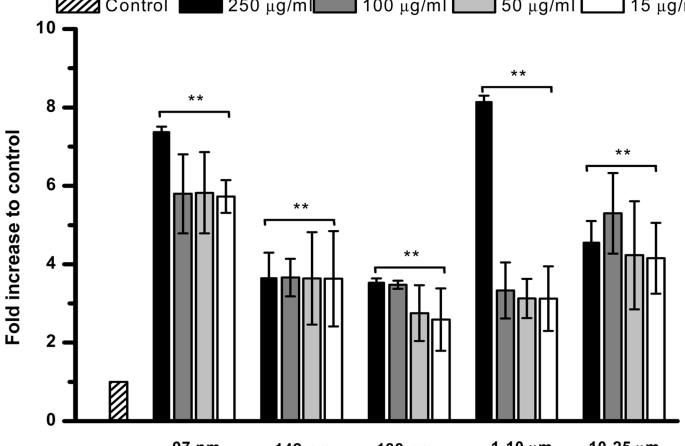

Figure 2. Cytotoxicity, oxidative stress, and inflammatory responses to THCPSi particles in Caco-2 and RAW 264.7 macrophage cells. Cellular viability of Caco-2 (a) cells and RAW 264.7 macrophage (b) cells after $24 \mathrm{~h}$ incubation with different concentrations ( $\mu \mathrm{g} / \mathrm{mL}$ ) of THCPSi particles, assessed by a luminescence assay. Intracellular ROS assessment of Caco-2 cells (c) and RAW 264.7 macrophage (d) cells after $24 \mathrm{~h}$ incubation with different concentrations $(\mu \mathrm{g} / \mathrm{mL})$ of THCPSi particles, determined with fluorescent $2^{\prime}, 7^{\prime}$-dichlorodihydrofluorescein diacetate (DCF-DA). A cell-free assay was performed to assess the interference of the particles in the fluorescence measured in the ROS assay. There was no evidence of significant interference from the particles. The negative control for ROS (cells only) was found to yield a value of $6.6 \% \pm 0.8$. TNF- $\alpha$ assessment of RAW 264.7 macrophage (e) cells after $24 \mathrm{~h}$ incubation with different concentrations of THCPSi particles. Error bars represent mean \pm SEM ( $n \geq 3$ ), except for TNF- $\alpha$, in which error bars represent s.d. for a given exposure group. Statistically there is a significant difference between untreated (control) cells incubated with buffer only (a, b, and e) or $0.09 \% \mathrm{H}_{2} \mathrm{O}_{2}$ (c and $\mathrm{d}$ ); ${ }^{*} P<0.05$ and ${ }^{*} P<0.01$.
The extent of the particle internalization by the cells was confirmed by confocal microscopy (Figure 3c) and by analyzing the different focal planes acquired through each sample (z-stacks). It was observed that the majority of the particles remained attached to the cell surface when compared to controls (Figure 3c). Furthermore, permeability of FITC-labeled THCPSi nanoparticles across Caco-2 monolayers indicated that less than $2 \%$ permeated across the Caco- 2 monolayers in $4.5 \mathrm{~h}$ regardless of the particle concentration (Figure $3 d$ ). We postulate that the negative $\zeta$-potential of $\mathrm{TH}$ CPSi particles (Figure 1d) together with their hydrophobic nature favors a strong particle-membrane interaction, ${ }^{31}$ but extensive internalization is prevented. ${ }^{32}$ The strong membrane interaction due to the THCPSi hydrophobic nature could cause toxicity, ${ }^{31}$ an effect not observed with our $142 \mathrm{~nm}$ THCPSi particles, which indicates their high biocompatibility.

In Vivo Distribution. For evaluating the suitability of $\mathrm{TH}-$ CPSi nanoparticle carriers for drug delivery applications, it is necessary to gain detailed knowledge about their biodistribution and behavior in vivo. The FITC-label is quenched in acidic environments such as the stomach and is, therefore, unsuitable for labeling particles intended for oral administration. To overcome this problem and assess the biodistribution of THCPSi nanoparticles in rats, we developed novel ${ }^{18} \mathrm{~F}$-labeled THCPSi nanoparticles. The $109.8 \mathrm{~min}$ half-life of ${ }^{18} \mathrm{~F}$ is long enough to allow the follow-up of slower physiological processes, such as passage in the gastrointestinal tract. Furthermore, as ${ }^{18} \mathrm{~F}$ is a short-lived positron emitter, it can be used for noninvasive visualization of particle distribution with positron emission tomography (PET), a fully translational, highly sensitive, and quantitative molecular imaging method.

Radiolabeled ${ }^{18} \mathrm{~F}$-THCPSi nanoparticles were administered to male Wistar rats using three different routes: oral administration by intragastric gavage, subcutaneous and intravenous injections. Control animals received ${ }^{18} \mathrm{~F}-\mathrm{NaF}$ in the vehicle to establish the biodistribution pattern of free $\left[{ }^{18} \mathrm{~F}\right]$ fluoride in the experimental setup. Administered doses are given in Table 1. The $\left[{ }^{18} \mathrm{~F}\right]$ fluoride ion is a known bone-seeking radiotracer, showing high and rapid bone uptake in rats. ${ }^{33,34}$ In the biodistribution studies with ${ }^{18} \mathrm{~F}$-labeled radiopharmaceuticals, ${ }^{18} \mathrm{~F}$ accumulation in bone is commonly regarded as an indicator of the level of defluorination of the tracer in vivo. ${ }^{33}$ Therefore, we administered to control animals $\left[{ }^{18} \mathrm{~F}\right] \mathrm{F}^{-}$as sodium fluoride to differentiate the ${ }^{18} \mathrm{~F}$-labeled nanoparticles from detached free ${ }^{18} \mathrm{~F}$ label in the nanoparticle biodistribution study. The results showed that the nanoparticles did not cross the intestinal wall, as seen by the negligible amount of radioactivity detected in the systemic circulation or organs outside the gastrointestinal tract (Figure 4a,b).

The observed appearance of radioactivity in bone and urine $(<0.6 \mathrm{ID} \% / \mathrm{g})$ at later time points was due to $\mathrm{mi}-$ 


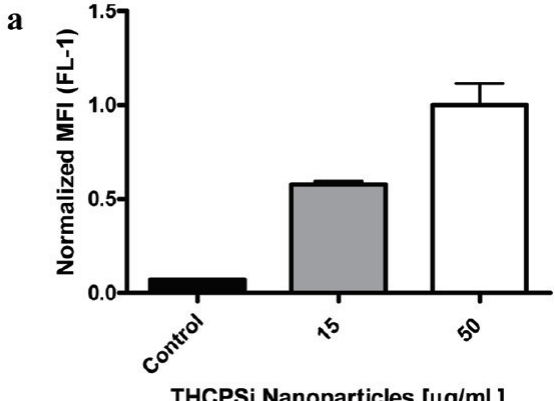

THCPSi Nanoparticles $[\mu \mathrm{g} / \mathrm{ml}]$

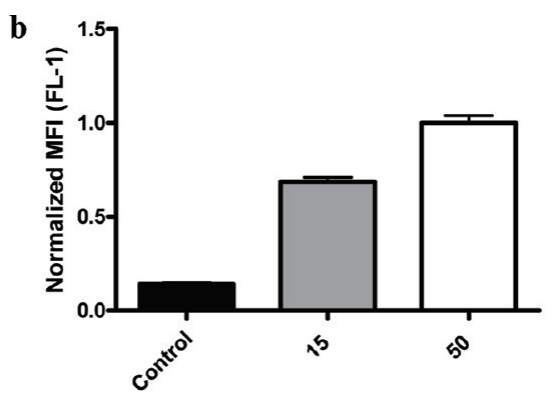

THCPSi Nanoparticles $[\mu \mathrm{g} / \mathrm{ml}]$

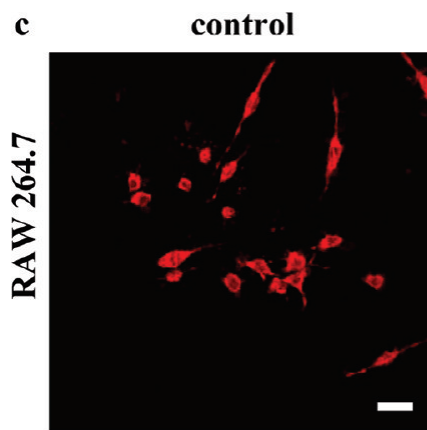

FITC-THCPSi
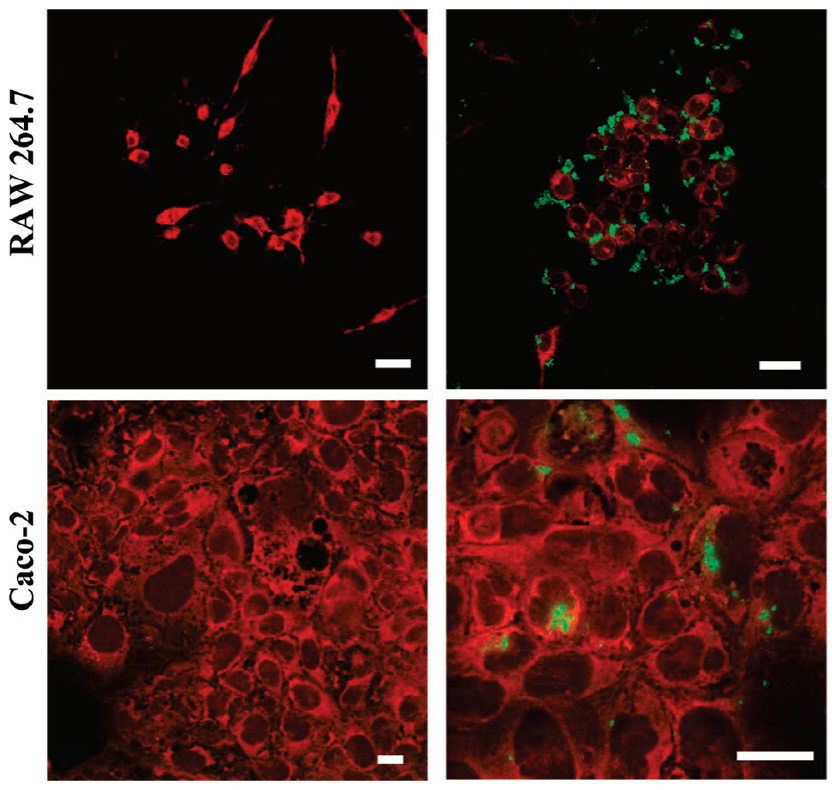

RAW 264.7 magnification

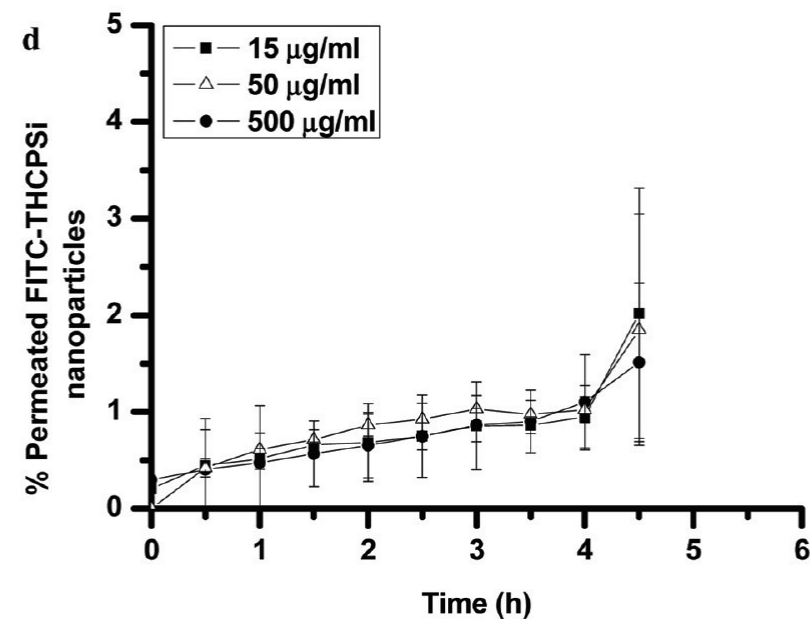

Figure 3. Particle association of FITC-labeled THCPSi nanoparticles in Caco-2 and RAW 264.7 macrophage cells. Caco-2 (a) and RAW 264.7 macrophage (b) cells were incubated with THCPSi nanoparticles (15 and $50 \mu \mathrm{g} / \mathrm{mL}$ ) for $3 \mathrm{~h}$. Untreated cells (control) are shown for comparison. FITC-labeled THCPSi nanoparticles (green) associated with cells were detected by flow cytometry. Mean fluorescence intensities (MFI) of FITC were measured and the values were normalized to the maximum extent of association in each cell line ( $n \geq 3$; mean \pm SEM). Confocal microscopy images, with $z$-stack planes of Caco-2 monolayers and RAW264.7 macrophage (c) cells (scale bars $=20 \mu \mathrm{m}$ ) stained in orange, were incubated for $3 \mathrm{~h}$ with FITC-labeled THCPSi nanoparticles (in green). The enlarged view represents a RAW 264.7 macrophage (scale bar $=5 \mu \mathrm{m}$ ). Permeation of FITC-labeled THCPSi nanoparticles across Caco- 2 cell monolayers (d) as a function of time ( $n \geq 3$; mean \pm SEM).

nor detachment of the ${ }^{18} \mathrm{~F}$-label, which can be caused by the in vivo defluorination or disintegration of the particles themselves. Distribution of the released radiolabel was consistent with the behavior of free ${ }^{18} \mathrm{~F}$ observed in control animals after administration of ${ }^{18} \mathrm{~F}-\mathrm{NaF}$ in the vehicle (Figure 4c,d). Macroautoradiographical analysis of the 


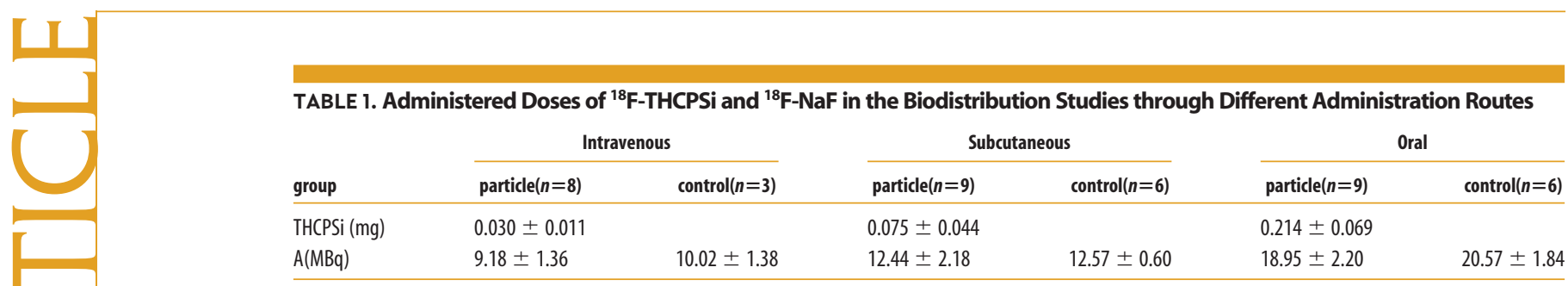

lower gastrointestinal tract showed that the majority of the administered nanoparticles reached the distal parts of colon in $6 \mathrm{~h}$ and were excreted in fecal pellets (Figure $5 a)$, but a more dispersed pattern was observed in control animals given ${ }^{18} \mathrm{~F}-\mathrm{NaF}$ (Figure $5 \mathrm{~b}$ ). Some interindividual variation associated with the activities of the animal (e.g., rest, drinking, coprophagy, and pica) during the study period was observed in the rate at which the particles were excreted. In some animals, reappearance of radioactivity in the small intestine was seen due to coprophagy (Figure 4b,d).

After intravenous administration into the lateral tail vein (Figure 6a) the particles were found in the liver and spleen, indicating that they are rapidly removed from the circulation. ${ }^{8}$ The tail segment surrounding the injection site was removed after sacrifice and its radioactivity counted to verify successful injection and lack of radioactivity at the injection site (data not shown). THCPSi nano- particles were administered subcutaneously under the loose skin at the back of the animal. The particles resided in the subcutaneous space for up to $4 \mathrm{~h}$ postinjection. This was confirmed by qualitative post-mortem autoradiography of the backside of the whole rat. The biodistribution data for subcutaneous administration showed only minimal leakage of dissociated ${ }^{18} \mathrm{~F}$-label into the circulation and subsequent accumulation in bone and urine (Figure $6 \mathrm{~b})$. As expected, significantly higher ${ }^{18} \mathrm{~F}$ bone uptake was seen in control animals in all administration routes. In addition, free ${ }^{18} \mathrm{~F}$ was rapidly extracted into urine in large amounts after intravenous and subcutaneous injections, whereas in particle-dosed animals less than 1 ID\%/g was excreted via the kidneys (Figure 4a). In control animals dosed orally, slightly slower kinetics of bone uptake were observed compared to the parenteral routes (Figure 4c), and macroautoradiography showed a dispersed distribution pattern, reflecting ${ }^{18} \mathrm{~F}$ absorption from the small in-
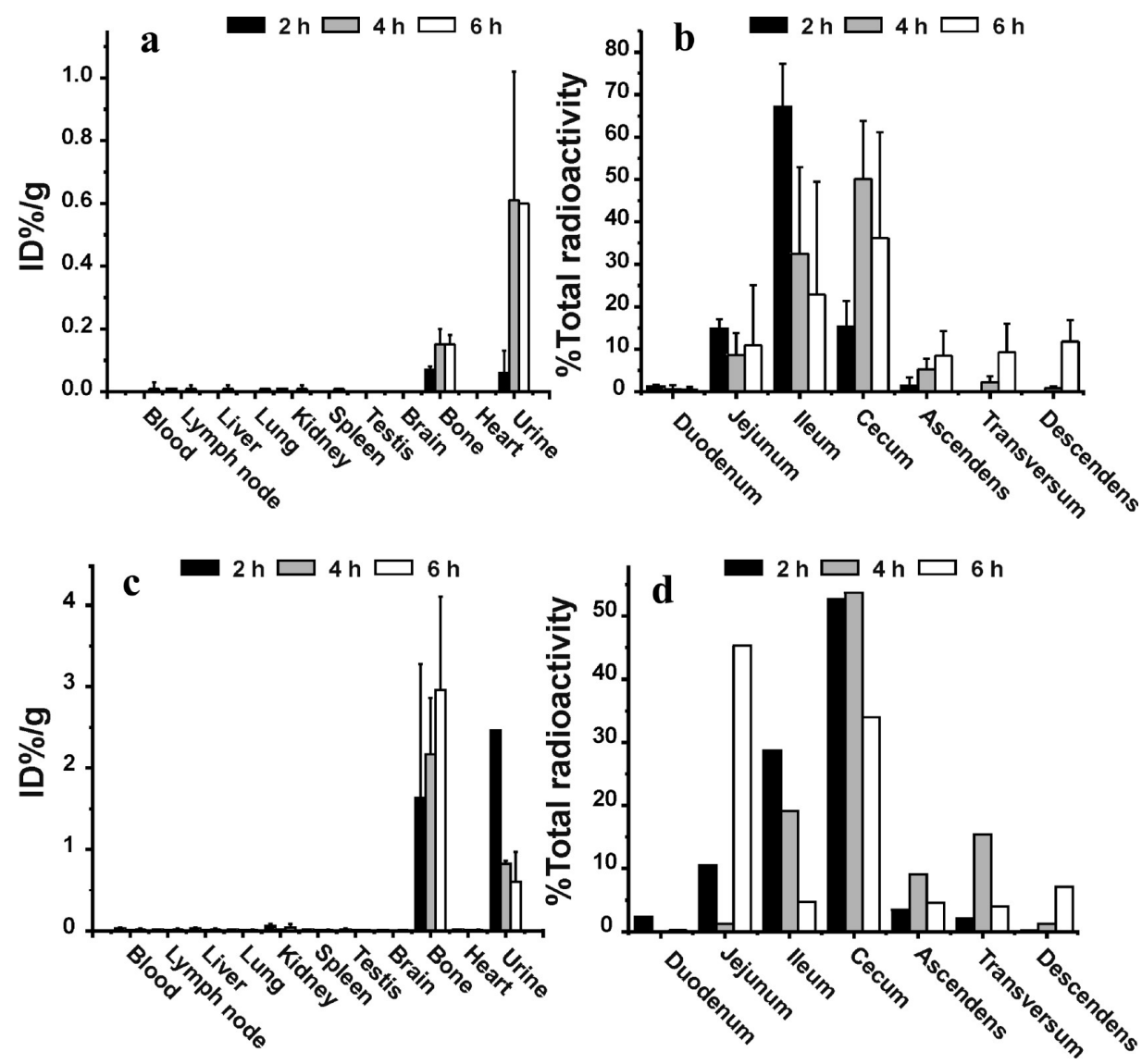

Figure 4. Biodistribution of ${ }^{18} \mathrm{~F}$-labeled THCPSi nanoparticles after oral administration (a) and their radioactive distribution in the lower $\mathrm{Gl}$ tract (b), as quantified from autoradiography ( $n=3-4$ at each time point; mean \pm s.d.). Biodistribution of free ${ }^{18} \mathrm{~F}-\mathrm{NaF}$ after oral administration in control animals (c) $(n=2$ at each time point; mean \pm s.d.) and its radioactive distribution in the lower GI tract in control animals after oral administration (d) quantified from autoradiography. 
a

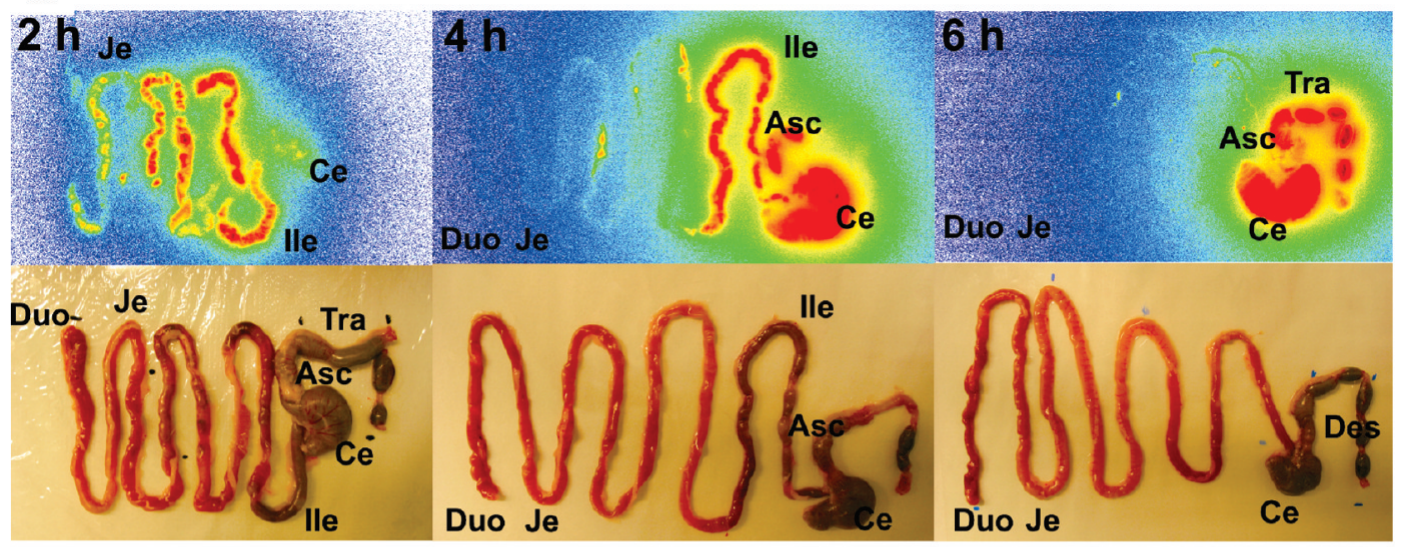

b

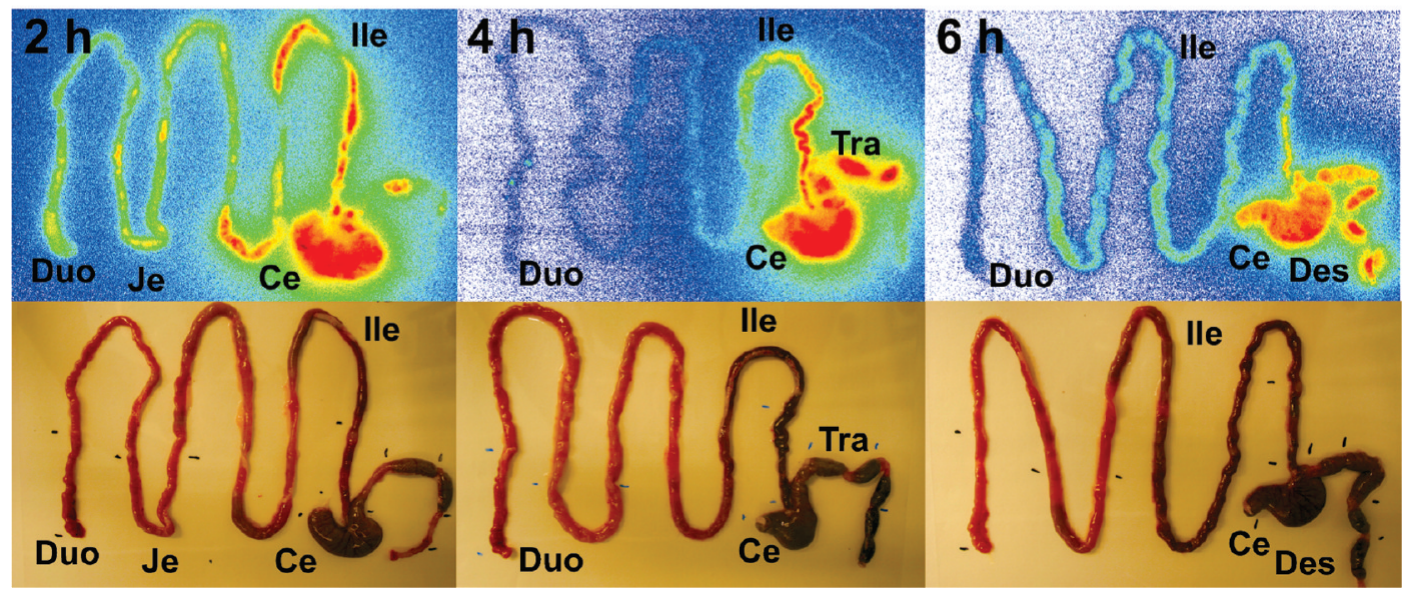

Figure 5. Macroautoradiographs and respective photographs of the Gl tracts of rats 2, 4, and $6 \mathrm{~h}$ (from left to right) after oral administration of ${ }^{18} \mathrm{~F}$-labeled THCPSi nanoparticles (a) and free ${ }^{18} \mathrm{~F}-\mathrm{NaF}$ (b). Abbreviations denote Duo, duodenum; Je, jejunum; Ile, ileum; Ce, cecum; Asc, ascendens; Tra, transversum; and Des, descendens.

testine as reported previously ${ }^{34}$ (Figure $\left.5 b\right)$. Biodistribution of free ${ }^{18} \mathrm{~F}-\mathrm{NaF}$ (Figure $6 \mathrm{c}, \mathrm{d}$ ) showed a pattern clearly distinguishable from that seen in particle-dosed animals (Figure $6 \mathrm{a}, \mathrm{b})$, demonstrating excellent in vivo stability of ${ }^{18} \mathrm{~F}$-THCPSi nanoparticles under different physiological conditions.

\section{CONCLUSION}

The THCPSi particles have been shown to cause different cellular responses depending on the cell type and route of administration, and therefore, particles with well-defined sizes were investigated in this study. The stability of THCPSi particles under the experimental timeline investigated and the ease of labeling them with fluorescent or radioactive labels for imaging pur- poses render the material a flexible platform for functionalization and further development in drug delivery applications. However, while the stability of THCPSi particles is prospective for their oral delivery, demonstrated biodegradability and favorable rate of degradation are critical for their subcutaneous administration and still need to be investigated. In conclusion, this study demonstrates for the first time the feasibility of oral delivery of silicon-based nanoparticles by means of in vitro cellular responses and interactions, as well as in vivo biodistribution, stability, and safety. The results presented here constitute the first quantitative analysis of the behavior of orally administered THCPSi nanoparticles compared with other delivery routes in rats.

\section{MATERIALS AND METHODS}

Preparation of Thermally Hydrocarbonized Porous Silicon (THCPSi) Particles. The free-standing porous silicon (PSi) films were anodized using single crystal silicon wafers (100) of $p$ +-type with resistivity values of $0.01-0.02 \Omega \mathrm{cm}$. The PSi was prepared by anodizing the wafers in an HF (38\%)-ethanol mixture. The free- standing films for microparticle production were etched for 40 min using a constant etching current $\left(50 \mathrm{~mA} / \mathrm{cm}^{2}\right)$.

In the case of THCPSi nanoparticle production, the method consisted of three different current pulses so that the first low current pulse is used to produce a similar mesoporous silicon structure as in the microparticles (porosity $=65 \%$ ). The second 


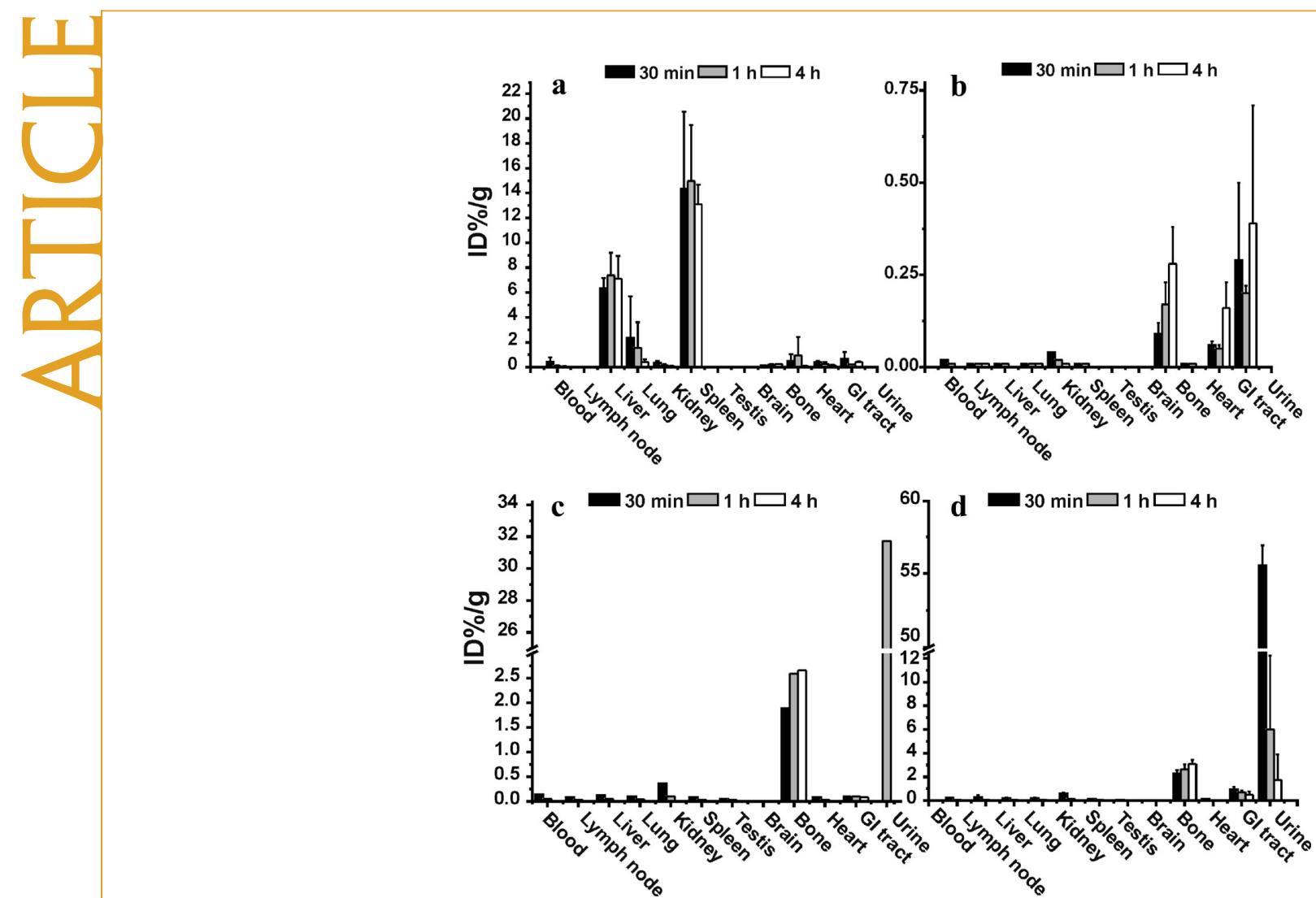

Figure 6. Biodistribution of ${ }^{18} \mathrm{~F}$-labeled THCPSi nanoparticles ( $a$ and $b ; n=2-4$ at each time point; $m e a n \pm s . d$.), and free ${ }^{18} \mathrm{~F}$-fluoride in control animals ( $c$ and d) after intravenous ( $a$ and $c$ ) and subcutaneous ( $b$ and d) administration ( $n=1-2$ at each time point; mean \pm s.d.).

pulse is shorter, with a high current pulse near the electropolishing region, which produces highly porous and mechanically fragile layers. The fragile layers predetermine the fracture sites on the plane parallel to the surface of the porous film (see Supporting Information, Figure 3), while the pores determine them on the perpendicular planes. A third zero-current pulse is then used to remove the possible electrolyte concentration gradients in the pores formed during the high current pulse, before the new etching cycle starts. After drying, the pulse etched freestanding films were thermally hydrocarbonized under a $\mathrm{N}_{2}$ /acetylene (1:1) flow at $500{ }^{\circ} \mathrm{C}$ for $10 \mathrm{~min}$ as described previously. ${ }^{3,19}$

The size reduction of the hydrocarbonized free-standing PS films (THCPSi) was done with ball milling. Wet milling was used in nanoparticle production in order to increase the grinding effcacy. Although the external surface of the milled nanoparticles is mainly composed of the initial hydrocarbonized pore walls ( $80-90 \%$ of the external surface), we tried to reduce oxidation of the new external surfaces created during the milling by using 1-decene as the milling liquid. The high grinding forces during ball milling raise the temperature of the suspension locally at the impact site, where it is high enough for a thermal hydrosilylation of aliphatic alkenes on the new surfaces ${ }^{35}$ but too low to oxidize the hydrocarbonized surfaces. The surface chemistry of particles was verified with FTIR (data not shown) and by testing the hydrophobicity of the nanoparticles. The final size separation and changes of the suspension media were done with centrifugation.

The microparticles were produced with a short dry milling of as-anodized free-standing films, after which the particles were wet-sieved or filtrated to the desired size fractions and the surface oxide was removed with a HF treatment prior to the hydrocarbonization treatment similar to that used for nanoparticles.

Particle Characterization. The pore volume, average pore diameter, and specific surface area of THCPSi micro- and nanoparticles were calculated from desorption branch of nitrogen sorption (Tristar 3000, Micromeritics Inc.) measurements (results not shown). Although the surface area and pore volume are reduced in the nanoparticles, they are still sufficient considering drug de- livery applications. The average nanoparticle sizes were measured from TEM (FEI Tecnai F12, Philips Electron Optics) images. The samples for TEM were prepared by adding droplets of the THCPSi nanoparticles (labeled and unlabeled) suspensions (prepared in HBSS, $\mathrm{pH}=7.4$ ) on the copper-carbon grids and allowing them to dry in a drybox for about $24 \mathrm{~h}$. The size distribution was then estimated using free ImageJ software. The microparticle size was determined with laser diffraction (Mastersizer, Malvern Instruments Ltd.) or with an automated optical microscopy analysis (Morphologi G3, Malvern Instruments Ltd.).

${ }^{18}$ F-Radiolabeling of THCPSi Nanoparticles. All reagents and $1 \times$ HBSS buffer were purchased from Sigma-Aldrich, except for absolute ethanol (Etax Aa, $\geq 99.7$ vol-\%, Altia Corporation) and polyethylene glycol-15-hydroxystearate solubilizer (Solutol HS 15 , BASF SE). Fluorine- 18 was produced in $a^{18} \mathrm{O}(\mathrm{p}, \mathrm{n})^{18} \mathrm{~F}$ reaction on a IBA Cyclone 5/10 cyclotron (Laboratory of Radiochemistry, University of Helsinki, Helsinki, Finland). To $1 \mathrm{mg}$ of THCPSi nanoparticles suspended in anhydrous DMF were added $500 \mu \mathrm{L}$ of $4 \%(\mathrm{v} / \mathrm{v})$ acetic acid and $0.5-1 \mathrm{GBq}$ of fluorine-18 as $\left[{ }^{[8} \mathrm{F}\right] \mathrm{KF} /$ Kryptofix 2.2.2 in $100 \mu \mathrm{L}$ of DMF. ${ }^{36,37}$ The solution was heated at $120^{\circ} \mathrm{C}$ for $15 \mathrm{~min}$. Radiolabeled nanoparticles were purified with sequential washes in absolute ethanol, ultrapure water, and $1 \times$ HBSS, $\mathrm{pH}=7.4$. Particles were centrifuged at $29000 \mathrm{~g}$, the supernatant was changed, and the particles were resuspended by sonication between washes. The final formulation was prepared in $5 \%$ Solutol- $1 \times$ HBSS $(\mathrm{pH}=7.4)$. Product specific radioactivity was $207 \pm 139 \mathrm{MBq} / \mathrm{mg}$ at administration. The $\zeta$-potential measurements were performed for each radiolabeled batch to verify that the THCPSi surface chemistry was not affected by the labeling reaction.

Cell Culture and Toxicity. The in vitro studies with the THCPSi particles were performed with human colon carcinoma (Caco-2, ATCC) and RAW 264.7 murine macrophage cells. Both cell lines were obtained from American Type Culture Collection (ATCC). The cells were cultured in $75 \mathrm{~cm}^{2}$ culture flasks (Corning Incorporated Life Sciences) using Dulbecco's modified Eagle's medium (DMEM, Euroclone S.p.A) supplemented with $10 \%$ fetal bovine serum (FBS, Gibco), 1\% nonessential amino acids, 1\% 
L-glutamine, penicillin (100 IU/mL), and streptomycin (100 $\mu \mathrm{g} /$ $\mathrm{mL}$; all from Euroclone S.p.A.). The culture was maintained in a BB 16 gas incubator at $37^{\circ} \mathrm{C}$ (Heraeus Instruments $\mathrm{GmbH}$ ) in an atmosphere of $5 \% \mathrm{CO}_{2}$ and $95 \%$ relative humidity. The growth medium was changed every other day until the time of use. The Caco-2 cells from passage numbers 31-40 and RAW 264.7 macrophages from passages $17-20$ were used in the experiments. Prior to each test, the RAW 264.7 macrophages were harvested using phosphate buffered saline (PBS)-ethylenediamine tetraacetic acid (EDTA) and Caco-2 cells were harvested using $0.25 \%$ $(\mathrm{v} / \mathrm{v})$ trypsin-EDTA-PBS. For the toxicity assessment, $2 \times 10^{4}$ cells in DMEM were seeded in 96-well plates and allowed to attach overnight. The medium was then aspirated, and $100 \mu \mathrm{L}$ of THCPSi particle suspensions with concentrations of 250, 100, 50 and $15 \mu \mathrm{g} / \mathrm{mL}$ were added to the wells. After $24 \mathrm{~h}, 100 \mu \mathrm{L}$ of the CellTiter-Glo reagent (Promega Corporation) was added to each well according to the manufacturer's instructions. In this assay the number of viable cells in culture is quantified based on the amount of ATP produced by metabolically active cells. Thus, the amount of ATP produced is directly proportional to the number of living cells presented in the culture. The plate was then measured for luminescence using a Varioskan Flash fluorometer (Thermo Fisher Scientific). All assays were made at least in triplicate. The TNF- $\alpha$ and ROS measurements were also made according to manufacturer's instructions.

Reactive Oxygen Species (ROS) Determination. The experiments were performed as described elsewhere. ${ }^{4,38}$ Briefly, in 96-well plates, $2 \times 10^{4}$ cells were seeded and allowed to attach overnight. The medium was then aspirated, and afterward, $100 \mu \mathrm{L}$ of $10 \mu \mathrm{M} \mathrm{2} 2^{\prime}, 7^{\prime}$-dichlorofluorescin diacetate (DCF-DA) solution was added and allowed to incubate $1 \mathrm{~h}$ at $37^{\circ} \mathrm{C}$. The solution was subsequently washed with HBSS and THCPSi suspension solutions of concentrations of $250,100,50$, and $15 \mu \mathrm{g} / \mathrm{mL}$ were added to the wells. Hydrogen peroxide treated cells $\left(\mathrm{H}_{2} \mathrm{O}_{2}\right.$ $0.09 \%$ ) was used as positive control. After treatment for $24 \mathrm{~h}$, the plate wells were washed and DCF fluorescence was measured in a Varioskan Flash (Thermo Fisher Scientific). Excitation and emission wavelengths were 498 and $522 \mathrm{~nm}$, respectively.

TNF- $\alpha$ Assay. To assess TNF- $\alpha$ production by the macrophages, an enzyme-linked immunoabsorbent assay (ELISA) was performed using a commercially available kit (Mouse TNF- $\alpha$ ELISA, Bender MedSystems GmbH). First, serial dilutions of murine TNF- $\alpha$ was prepared and pipetted into the 96-well plates precoated with immobilized antibodies. Precollected supernatants from all the treated and control cells were then added to the wells. After $2 \mathrm{~h}$, the wells were washed and biotinconjugated specific for mouse TNF- $\alpha$ was added to the wells. The wells were then completely washed to remove any unbound reactants after another $2 \mathrm{~h}$. The amount of TNF- $\alpha$ present in each well was determined by measuring the absorbance for each sample in a fluorometer at $450 \mathrm{~nm}$. The amount of TNF- $\alpha$ produced by the RAW 264.7 macrophage cells was then calculated from the standard curve of known amounts of murine TNF- $\alpha$. It should be emphasized that TNF- $\alpha$ does bind to THCPSi particles. Therefore, we have performed TNF- $\alpha$ binding experiments under the same conditions used during cell exposure in order to evaluate the existing of binding and to further correct the results as described elsewhere. ${ }^{39}$

Flow Cytometry. In six-well plates (Corning Incorporated Life Sciences), Caco-2 or RAW 264.7 macrophage suspensions were seeded in every individual well and allowed to attach overnight. Caco-2 and RAW 264.7 cells were incubated with 15 or $50 \mu \mathrm{g} / \mathrm{mL}$ of FITC-labeled THCPSi nanoparticle solutions for $3 \mathrm{~h}$. After washing with HBSS, the cells in the wells were detached with trypsinEDTA. The cells were then carefully collected and centrifuged. The final washing was performed with PBS-EDTA to prevent aggregation of the cells. The cells were then fixed with $4 \%$ paraformaldehyde (PFA, Fluka) in PBS for $20 \mathrm{~min}$. The samples were analyzed by a BD LSR II flow cytometer (BD Biosciences) with a laser excitation wavelength of $488 \mathrm{~nm}$, using FACSDiva software. Exactly 10000 events were recorded. Untreated cells were used as controls and the background (due to the particles only) and debris were subtracted.

Caco-2 Permeability Experiments. Transport experiments were performed in $\mathrm{HBSS} \mathrm{pH} 7.4$ at $37^{\circ} \mathrm{C}$ using an orbital shaking (50 rpm). The Caco-2 cells were seeded on 12-Transwell cell culture inserts (polycarbonate membranes, pore size $3 \mu \mathrm{m}$, growth area $0.33 \mathrm{~cm}^{2}$, Corning Costar Corp). The cells were kept under the same conditions as described in Cell Culture and Toxicity. Cells were used in the experiments $21-28$ days after seeding. The permeability of FITC-labeled THCPSi nanoparticles was studied across Caco-2 cell monolayers in an apical-to-basolateral direction for $4.5 \mathrm{~h}$. Control experiments (untreated cells) were performed in parallel. The amount of FITC-labeled THCPSi nanoparticles that had permeated across the Caco- 2 monolayers was then calculated from a standard curve of known FITC-labeled THCPSi nanoparticles determined previously. The FITC-labeled nanoparticles were stable under experimental conditions described and no free FITC was detected during the study. The monolayer integrity after each experiment was determined by transepithelial electrical resistance and further assessed after the permeability tests with $\left[{ }^{14} \mathrm{C}\right]$ mannitol (Amersham Biosciences). Under the experimental conditions used, no damage to the Caco-2 monolayers was observed (data not shown), which supports the cytotoxicity results in the study.

Confocal Microscopy. A solution of $1 \times 10^{7}$ cells $/ \mathrm{mL}$ was seeded in 8-well chambered No. 1.0 borosilicate coverglass system plates (Lab-Tek Nunc) and allowed to attach overnight. After that, 30 or $100 \mu \mathrm{g} / \mathrm{mL}$ of FITC-labeled THCPSi nanoparticles suspensions were added to the wells for a final concentration of 15 and $50 \mu \mathrm{g} / \mathrm{mL}$ and incubated for $3 \mathrm{~h}$. The particles were then removed and the wells washed three times with HBSS. CellMask (Invitrogen) was added for cell staining according to manufacturer specifications. Prior to measurements, the cells were fixed with $4 \%$ paraformaldehyde (PFA, Fluka) in PBS for $20 \mathrm{~min}$. Confocal pictures were taken with a Leica SP2 inverted confocal microscope, equipped with argon (488 nm) and DPSS (561 nm) lasers, and using a HCX Plan Apochromat $63 \times / 1.2$ water immersion objective (Leica Microsystems).

In vivo Biodistribution. All animal experiments were approved by the National Committee for Animal Experimentation in Finland (State Provincial Office of Southern Finland, Hämeenlinna). The ${ }^{18} \mathrm{~F}$-labeled THCPSi nanoparticles were administered to male Wistar Han rats (age 7-10 weeks, University of Helsinki Laboratory Animal Centre) in $5 \%$ Solutol-1 $\times$ HBSS $(\mathrm{pH}=7.4)$ either by intragastric gavage, subcutaneously on the back, or intravenously via the lateral tail vein $(n=8-9$ for each administration route). Control animals ( $n=3-6$ for each route) received ${ }^{18} \mathrm{~F}-\mathrm{NaF}$ in $5 \%$ Solutol- $1 \times$ HBSS. Animals were sacrificed at 2, 4, and $6 \mathrm{~h}$, or at $30 \mathrm{~min}, 1$, and $4 \mathrm{~h}$ after administration for enteral and parenteral routes, respectively.

Samples from blood, urine, mesenteric lymph node, liver, lung, kidney, spleen, testis, brain, bone, and stomach were collected. For intestinal biodistribution of radioactivity, the lower gastrointestinal tract was excised and samples were taken from duodenum, jejunum, ileum, cecum, and the ascending, transverse, and descending parts of colon. Samples were weighed and their radioactivity measured with a gamma counter (Wizard 3, PerkinElmer).

Macroautoradiography of the Lower Gastrointestinal Tract. For animals dosed by intragastric gavage, the whole lower gastrointestinal tract was imaged with digital autoradiography prior to sampling for radioactivity counting. The excised intestines were arranged on a Fujifilm BAS SR2040 imaging plate together with ${ }^{18} \mathrm{~F}$ standards and photographed for macroanatomical identification of different parts. Autoradiographs were analyzed with AIDA 2.0 imaging software (Raytest Isotopenmessgeräte $\mathrm{GmbH}$ ).

Acknowledgment. The financial support from Academy of Finland (Grant Nos. 127099, 123037, 122314, 115385), the Jenny and Antti Wihuri Foundation, and the University of Helsinki Research Funds is acknowledged. Dr. Kerttuli Helariutta is acknowledged for production of the ${ }^{18} \mathrm{~F}$ isotope. M.Sc. students Jakub Šimeček and Venla Turtiainen are thanked for skillful technical assistance with the animal studies.

Supporting Information Available: FTIR spectra of unlabeled and ${ }^{18} \mathrm{~F}$-labled THCPSi free-standing films, intracellular ROS response, and viability of Caco-2 cells incubated with several concentrations of silicic acid from the complete dissolution of TH- 
CPSi particles, silicic acid determination protocol, and crosssectional SEM picture of PSi layer. This material is available free of charge via the Internet at http://pubs.acs.org.

\section{REFERENCES AND NOTES}

1. Canham, L. Bioactive Silicon Structure Fabrication through Nanoetching Techniques. Adv. Mater. 1995, 7, 1033-1037.

2. Anglin, E. J.; Cheng, L.; Freeman, W. R.; Sailor, M. J. Porous Silicon in Drug Delivery Devices and Materials. Adv. Drug Delivery Rev. 2008, 60, 1266-1277.

3. Salonen, J.; Kaukonen, A. M.; Hirvonen, J.; Lehto, V. P. Mesoporous Silicon in Drug Delivery Applications. J. Pharm. Sci. 2008, 97, 632-653.

4. Santos, H. A.; Riikonen, J.; Salonen, J.; Makila, E.; Heikkila, T.; Laaksonen, T.; Peltonen, L.; Lehto, V. P.; Hirvonen, J. In Vitro Cytotoxicity of Porous Silicon Microparticles: Effect of the Particle Concentration, Surface Chemistry and Size. Acta Biomater. 2010, 6, 2721-2731.

5. Santini, J. T., Jr.; Cima, M. J.; Langer, R. A ControlledRelease Microchip. Nature 1999, 397, 335-358.

6. Lin, V. S.; Motesharei, K.; Dancil, K. P.; Sailor, M. J.; Ghadiri, M. R. A Porous Silicon-Based Optical Interferometric Biosensor. Science 1997, 278, 840-843.

7. Collins, R. T.; Fauchet, P. M. Porous Silicon: From Luminescence to LEDs. Phys. Today 1997, 50, 24-32.

8. Park, J. H.; Gu, L.; von Maltzahn, G.; Ruoslahti, E.; Bhatia, S. N.; Sailor, M. J. Biodegradable Luminescent Porous Silicon Nanoparticles for In Vivo Applications. Nat. Mater. 2009, 8, 331-336.

9. Low, S. P.; Voelcker, N. H.; Canham, L. T.; Williams, K. A. The Biocompatibility of Porous Silicon in Tissues of the Eye. Biomaterials 2009, 30, 2873-2880.

10. Cheng, L.; Anglin, E.; Cunin, F.; Kim, D.; Sailor, M. J.; Falkenstein, l.; Tammewar, A.; Freeman, W. R. Intravitreal Properties of Porous Silicon Photonic Crystals: a Potential Self-Reporting Intraocular Drug-Delivery Vehicle. $\mathrm{Br} J$ Ophthalmol. 2008, 92, 705-711.

11. Brayden, D. J. Controlled Release Technologies for Drug Delivery. Drug Discovery Today 2003, 8, 976-978.

12. Salonen, J.; Laitinen, L.; Kaukonen, A. M.; Tuura, J.; Bjorkqvist, M.; Heikkila, T.; Vaha-Heikkila, K.; Hirvonen, J.; Lehto, V. P. Mesoporous Silicon Microparticles for Oral Drug Delivery: Loading and Release of Five Model Drugs. J. Controlled Release 2005, 108, 362-374.

13. Foraker, A. B.; Walczak, R. J.; Cohen, M. H.; Boiarski, T. A.; Grove, C. F.; Swaan, P. W. Microfabricated Porous Silicon Particles Enhance Paracellular Delivery of Insulin across Intestinal Caco-2 Cell Monolayers. Pharm. Res. 2003, 20, 110-116.

14. Kaukonen, A. M.; Laitinen, L.; Salonen, J.; Tuura, J.; Heikkila T.; Limnell, T.; Hirvonen, J.; Lehto, V. P. Enhanced In Vitro Permeation of Furosemide Loaded into Thermally Carbonized Mesoporous Silicon (TCPSi) Microparticles. Eur. J. Pharm. Biopharm. 2007, 66, 348-356.

15. Limnell, T.; Riikonen, J.; Salonen, J.; Kaukonen, A. M.; Laitinen, L.; Hirvonen, J.; Lehto, V. P. Surface Chemistry and Pore Size Affect Carrier Properties of Mesoporous Silicon Microparticles. Int. J. Pharm. 2007, 343, 141-147.

16. Wang, F.; Hui, H.; Barnes, T. J.; Barnett, C.; Prestidge, C. A. Oxidized Mesoporous Silicon Microparticles for Improved Oral Delivery of Poorly Soluble Drugs. Mol. Pharm. 2010, 7, 227-236.

17. Sudeep, P. K.; Page, Z.; Emrick, T. PEGylated Silicon Nanoparticles: Synthesis and Characterization. Chem. Commun. (Cambridge) 2008, 6126-6127.

18. Erogbogbo, F.; Yong, K. T.; Roy, I.; Xu, G.; Prasad, P. N.; Swihart, M. T. Biocompatible Luminescent Silicon Quantum Dots for Imaging of Cancer Cells. ACS Nano 2008, 2, 873-878.

19. Kilpelainen, M.; Riikonen, J.; Vlasova, M. A.; Huotari, A.; Lehto, V. P.; Salonen, J.; Herzig, K. H.; Jarvinen, K. In Vivo Delivery of a Peptide, Ghrelin Antagonist, with Mesoporous Silicon Microparticles. J. Controlled Release 2009, 137, 166-170.

20. Heinrich, J. L.; Curtis, C. L.; Credo, G. M.; Sailor, M. J.;
Kavanagh, K. L. Luminescent Colloidal Silicon Suspensions from Porous Silicon. Science 1992, 255, 66-68.

21. Nel, A.; Xia, T.; Madler, L.; Li, N. Toxic Potential of Materials at the Nanolevel. Science 2006, 311, 622-627.

22. Salonen, J.; Björkqvist, M.; Laine, E.; Niinistö, L. Stabilization of Porous Silicon Surface by Thermal Decomposition of Acetylene. Appl. Surf. Sci. 2004, 225, 389-394.

23. Cai, L.; Lu, S.; Pike, V. W. Chemistry with $\left[{ }^{18} \mathrm{~F}\right]$ Fluoride Ion. Eur. J. Org. Chem. 2008, 2008, 2853-2873.

24. Laaksonen, T.; et al. Failure of MTT as a Toxicity Testing Agent for Mesoporous Silicon Microparticles. Chem. Res. Toxicol. 2007, 20, 1913-1918.

25. AshaRani, P. V.; Low Kah Mun, G.; Hande, M. P.; Valiyaveettil, S. Cytotoxicity and Genotoxicity of Silver Nanoparticles in Human Cells. ACS Nano 2009, 3, 279-290.

26. Choi, J.; Zhang, Q.; Reipa, V.; Wang, N. S.; Stratmeyer, M. E.; Hitchins, V. M.; Goering, P. L. Comparison of Cytotoxic and Inflammatory Responses of Photoluminescent Silicon Nanoparticles with Silicon Micron-sized Particles in RAW 264.7 Macrophages. J. Appl. Toxicol. 2009, 29, 52-60.

27. Jiang, W.; Kim, B. Y.; Rutka, J. T.; Chan, W. C. NanoparticleMediated Cellular Response is Size-Dependent. Nat. Nanotechnol. 2008, 3, 145-150.

28. Rejman, J.; Oberle, V.; Zuhorn, I. S.; Hoekstra, D. SizeDependent Internalization of Particles via the Pathways of Clathrin- and Caveolae-Mediated Endocytosis. Biochem. J. 2004, 377, 159-169.

29. Chithrani, B. D.; Ghazani, A. A.; Chan, W. C. Determining the Size and Shape Dependence of Gold Nanoparticle Uptake into Mammalian Cells. Nano Lett. 2006, 6, 662-668.

30. Chapekar, M. S.; Zaremba, T. G.; Kuester, R. K.; Hitchins, V. M. Synergistic Induction of Tumor Necrosis Factor Alpha by Bacterial Lipopolysaccharide and Lipoteichoic Acid in Combination with Polytetrafluoroethylene Particles in a Murine Macrophage Cell Line RAW 264.7. J. Biomed. Mater. Res. 1996, 31, 251-256.

31. Tavares, J. C.; Cornelio, D. A.; da Silva, N. B.; de Moura, C. E.; de Queiroz, J. D.; Sa, J. C.; Alves, C., Jr.; de Medeiros, S. R. Effect of Titanium Surface Modified by Plasma Energy Source on Genotoxic Response In Vitro. Toxicology 2009, 262, 138-144.

32. Gratton, S. E.; Ropp, P. A.; Pohlhaus, P. D.; Luft, J. C.; Madden, V. J.; Napier, M. E.; DeSimone, J. M. The Effect of Particle Design on Cellular Internalization Pathways. Proc. Natl. Acad. Sci. U.S.A. 2008, 105, 11613-11618.

33. Schou, M.; et al. PET Evaluation of Novel Radiofluorinated Reboxetine Analogs as Norepinephrine Transporter Probes in the Monkey Brain. Synapse 2004, 53, 57-67.

34. Charkes, N. D.; Brookes, M.; Makler, P. T., Jr. Studies of Skeletal Tracer Kinetics: II. Evaluation of a FiveCompartment Model of $\left[{ }^{18} \mathrm{~F}\right]$ Fluoride Kinetics in Rats. J. Nucl. Med. 1979, 20, 1150-1157.

35. Boukherroub, R.; Morin, S.; Wayner, D. D. M.; Bensebaa, F.; Sproule, G. I.; Baribeau, J. M.; Lockwood, D. J. Ideal Passivation of Luminescent Porous Silicon by Thermal, Noncatalytic Reaction with Alkenes and Aldehydes. Chem. Mater. 2001, 13, 2002-2011.

36. Bohn, P.; Deyine, A.; Azzouz, R.; Bailly, L.; Fiol-Petit, C.; Bischoff, L.; Fruit, C.; Marsais, F.; Vera, P. Design of SiliconBased Misonidazole Analogues and $\left[{ }^{18}\right] \mathrm{F}$-Radiolabelling. Nucl. Med. Biol. 2009, 36, 895-905.

37. Mu, L.; et al. Silicon-Based Building Blocks for One-Step ${ }^{18}$ F-Radiolabeling of Peptides for PET Imaging. Angew. Chem., Int. Ed. 2008, 47, 4922-4925.

38. Heikkila, T.; Santos, H. A.; Kumar, N.; Murzin, D. Y.; Salonen, J.; Laaksonen, T.; Peltonen, L.; Hirvonen, J.; Lehto, V. P. Cytotoxicity Study of Ordered Mesoporous Silica MCM-41 and SBA-15 Microparticles on Caco-2 Cells. Eur. J. Pharm. Biopharm. 2010, 74, 483-494.

39. Kocbach, A.; Totlandsdal, A. I.; Lag, M.; Refsnes, M.; Schwarze, P. E. Differential Binding of Cytokines to Environmentally Relevant Particles: A Possible Source for Misinterpretation of In Vitro Results? Toxicol. Lett. 2008, 176, 131-137. 